\title{
Molybdenum Carbonyl Complexes with Citrate and its relevant Carboxylates
}

Motoki Takuma, Yasuhiro Ohki, and Kazuyuki Tatsumi*

\section{Supporting Information}

Table 1. Crystal Data for $\left(\mathrm{Et}_{4} \mathrm{~N}\right)_{3}\left[(\mathrm{Hcit}) \mathrm{Mo}(\mathrm{CO})_{3}\right] \quad(\mathbf{1}), \quad\left(\mathrm{Et}_{4} \mathrm{~N}\right)_{2}\left[(\mathrm{Hcmal}) \mathrm{Mo}(\mathrm{CO})_{3}\right] \quad$ (2), $\left(\mathrm{Et}_{4} \mathrm{~N}\right)_{2}\left[(\mathrm{Hmal}) \mathrm{Mo}(\mathrm{CO})_{3}\right](3)$, and $\left(\mathrm{Me}_{4} \mathrm{~N}\right)_{2}\left[(\mathrm{Meida}) \mathrm{Mo}(\mathrm{CO})_{3}\right](4)$

\begin{tabular}{lllll}
\hline & \multicolumn{1}{c}{$\mathbf{1}$} & \multicolumn{1}{c}{$\mathbf{2}$} & \multicolumn{1}{c}{$\mathbf{3}$} & \multicolumn{1}{c}{$\mathbf{4}$} \\
\hline formula & $\mathrm{C}_{33} \mathrm{H}_{65} \mathrm{~N}_{3} \mathrm{O}_{10} \mathrm{Mo}$ & $\mathrm{C}_{24} \mathrm{H}_{46} \mathrm{~N}_{2} \mathrm{O}_{8} \mathrm{Mo}$ & $\mathrm{C}_{23} \mathrm{H}_{44} \mathrm{~N}_{2} \mathrm{O}_{8} \mathrm{Mo}$ & $\mathrm{C}_{16} \mathrm{H}_{31} \mathrm{~N}_{3} \mathrm{O}_{7} \mathrm{Mo}$ \\
mol wt $\left(\mathrm{g} \mathrm{mol}^{-1}\right)$ & 759.83 & 586.58 & 572.55 & 473.38 \\
cryst syst & orthorhombic & orthorhombic & orthorhombic & orthorhombic \\
space group & $P 2_{1} 2_{1} 2_{1}(\mathrm{No} .19)$ & $P 2_{1} 2_{1} 2_{1}(\mathrm{No} .19)$ & $P 2_{1} 2_{1} 2_{1}(\mathrm{No} .19)$ & $P 2_{1} 2_{1} 2_{1}($ No. 19) \\
cryst color & yellow & yellow & yellow & yellow \\
a $(\AA)$ & $9.994(4)$ & $12.034(4)$ & $12.00(1)$ & $6.773(3)$ \\
b $(\AA)$ & $17.599(7)$ & $13.340(4)$ & $13.27(1)$ & $15.479(7)$ \\
c $(\AA)$ & $22.225(9)$ & $17.719(6)$ & $17.83(2)$ & $20.470(10)$ \\
V $\left(\AA \AA^{3}\right)$ & $3909(2)$ & $2844(1)$ & $2840(4)$ & $2146(1)$ \\
$\mathrm{Z}$ & 4 & 4 & 4 & 4 \\
$\rho_{\text {calcd }}\left(\mathrm{g} \mathrm{cm}{ }^{-3}\right)$ & 1.271 & 1.264 & 1.265 & 1.378 \\
$2 \theta_{\text {max }}($ deg $)$ & 55 & 55 & 55 & 55 \\
no. of unique rflns & 4936 & 3630 & 3525 & 3601 \\
no. of obsd rflns & 6480 & 4793 & 3390 & 2247 \\
no. of parameters & 424 & 236 & 307 & 245 \\
$\mathrm{R}$ & 0.035 & 0.082 & 0.062 & 0.036 \\
Rw & 0.036 & 0.127 & 0.067 & 0.040 \\
GOF & 1.27 & 2.90 & 2.02 & 1.52 \\
\hline
\end{tabular}

${ }^{a}$ Observation criterion $I>3 \mathrm{~s}(I) .{ }^{b} \mathrm{R}=\mathrm{S} \| F o|-| F c|| \mathrm{S}|F o| . \quad{ }^{c} \mathrm{Rw}=[\{\mathrm{S} w(|F o|-|F c|) 2\} / \mathrm{S} w F o 2] 1 / 2 .{ }^{d}$ $\mathrm{GOF}=[\{\mathrm{S} w(|F o|-|F c|) 2\} /(N o-N p)] 1 / 2$, where $N o$ and $N p$ denote the number of data and parameters. 


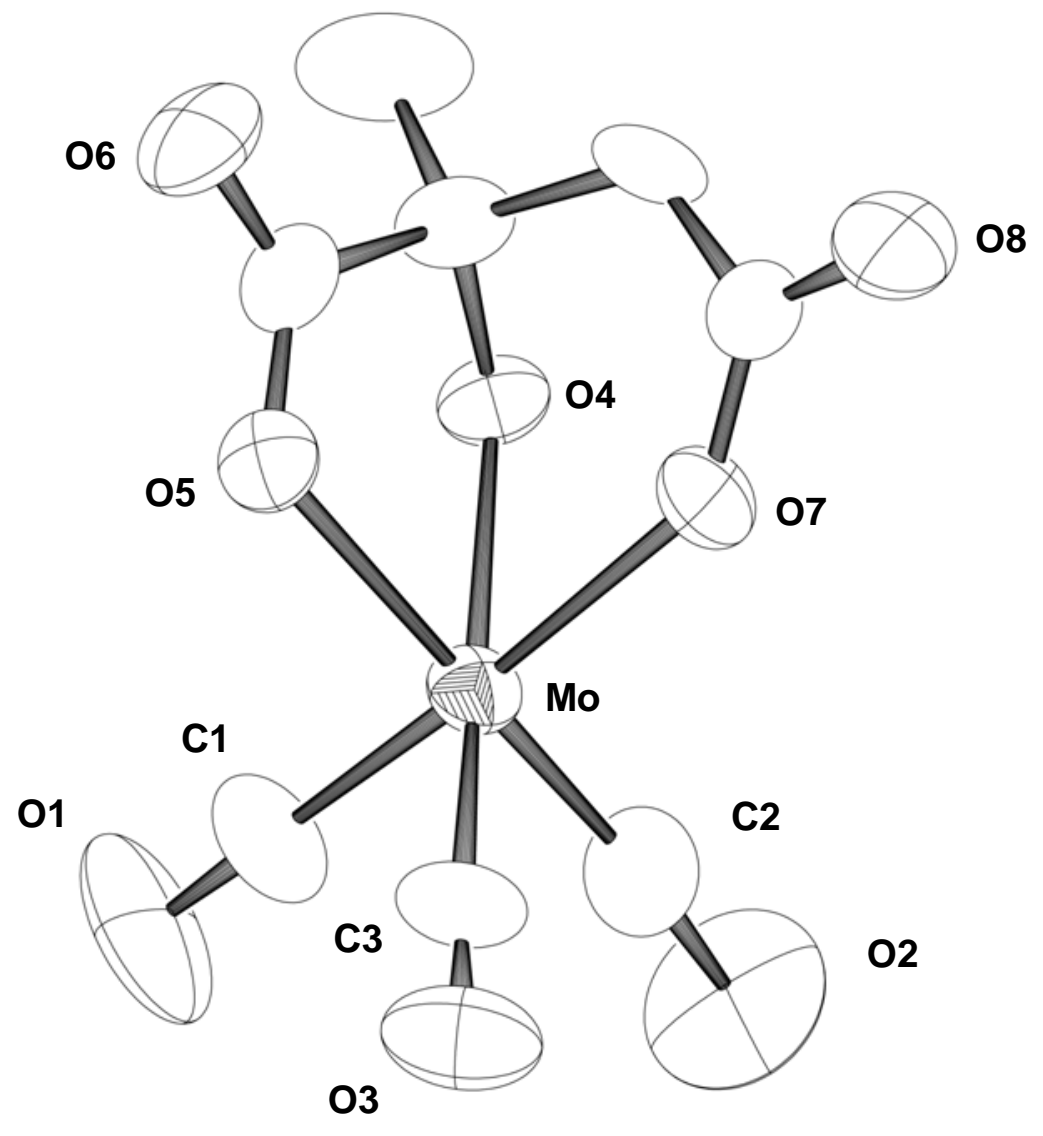

Figure S1. ORTEP drawing of the complex anion of $\left(\mathrm{Et}_{4} \mathrm{~N}\right)_{2}\left[(\mathrm{Hcmal}) \mathrm{Mo}(\mathrm{CO})_{3}\right](2)$. 
Table S1. Selected Bond Distances $(\AA)$ and Angles $(\mathrm{deg})$ in $\left(\mathrm{Et}_{4} \mathrm{~N}\right)_{2}\left[(\mathrm{Hcmal}) \mathrm{Mo}(\mathrm{CO})_{3}\right](3)$

\begin{tabular}{llll} 
& \multicolumn{3}{c}{ Bond Distances $(\AA)$} \\
$\mathrm{Mo}-\mathrm{C} 1$ & $1.91(1)$ & $\mathrm{Mo}-\mathrm{C} 2$ & $1.89(2)$ \\
$\mathrm{Mo}-\mathrm{C} 3$ & $2.02(2)$ & $\mathrm{Mo}-\mathrm{O} 4$ & $2.308(8)$ \\
$\mathrm{Mo}-\mathrm{O} 5$ & $2.219(8)$ & $\mathrm{Mo}-\mathrm{O} 7$ & $2.237(8)$
\end{tabular}

Bond Angles (deg)

$\begin{array}{llll}\mathrm{O} 4-\mathrm{Mo}-\mathrm{O} 5 & 71.4(3) & \mathrm{O} 4-\mathrm{Mo}-\mathrm{O} 7 & 75.4(3) \\ \mathrm{O} 5-\mathrm{Mo}-\mathrm{O} 7 & 81.1(3) & \mathrm{C} 1-\mathrm{Mo}-\mathrm{C} 2 & 80.1(7) \\ \mathrm{C} 1-\mathrm{Mo}-\mathrm{C} 3 & 85.2(7) & \mathrm{C} 2-\mathrm{Mo}-\mathrm{C} 3 & 83.9(7) \\ \mathrm{O} 4-\mathrm{Mo}-\mathrm{C} 1 & 101.7(6) & \mathrm{O} 4-\mathrm{Mo}-\mathrm{C} 2 & 102.5(6) \\ \mathrm{O} 4-\mathrm{Mo}-\mathrm{C} 3 & 171.2(4) & \mathrm{O} 5-\mathrm{Mo}-\mathrm{C} 1 & 97.0(5) \\ \mathrm{O} 5-\mathrm{Mo}-\mathrm{C} 2 & 172.8(7) & \mathrm{O} 5-\mathrm{Mo}-\mathrm{C} 3 & 102.6(4) \\ \mathrm{O} 7-\mathrm{Mo}-\mathrm{C} 1 & 176.9(7) & \mathrm{O} 7-\mathrm{Mo}-\mathrm{C} 2 & 101.4(6) \\ \mathrm{O} 7-\mathrm{Mo}-\mathrm{C} 3 & 97.6(5) & & \end{array}$




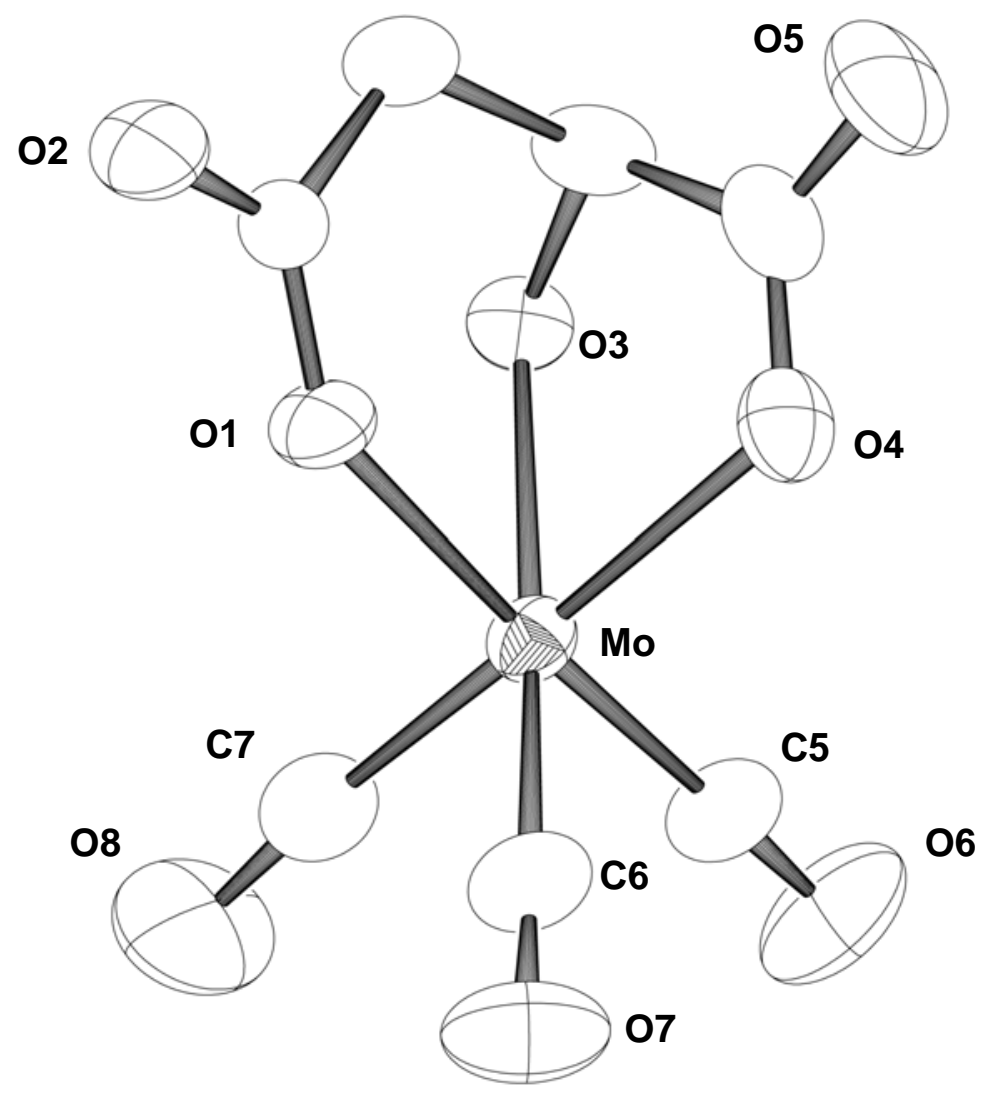

Figure S2. ORTEP drawing of the complex anion of $\left(\mathrm{Et}_{4} \mathrm{~N}\right)_{2}\left[(\mathrm{Hmal}) \mathrm{Mo}(\mathrm{CO})_{3}\right](3)$. 
Table S2. Selected Bond Distances $(\AA)$ and Angles $(\mathrm{deg})$ in $\left(\mathrm{Et}_{4} \mathrm{~N}\right)_{2}\left[(\mathrm{Hmal}) \mathrm{Mo}(\mathrm{CO})_{3}\right](\mathbf{3})$ Bond Distances $(\AA)$

$\begin{array}{llll}\mathrm{Mo}-\mathrm{C} 1 & 1.931(8) & \mathrm{Mo}-\mathrm{C} 2 & 1.939(4) \\ \mathrm{Mo}-\mathrm{C} 3 & 1.902(8) & \mathrm{Mo}-\mathrm{O} 4 & 2.281(5) \\ \mathrm{Mo}-\mathrm{O} 5 & 2.250(5) & \mathrm{Mo}-\mathrm{O} 7 & 2.254(5)\end{array}$

Bond Angles (deg)

$\begin{array}{llll}\mathrm{O} 4-\mathrm{Mo}-\mathrm{O} 5 & 73.0(2) & \mathrm{O} 4-\mathrm{Mo}-\mathrm{O} 7 & 75.7(2) \\ \mathrm{O} 5-\mathrm{Mo}-\mathrm{O} 7 & 80.7(2) & \mathrm{C} 1-\mathrm{Mo}-\mathrm{C} 2 & 83.4(4) \\ \mathrm{C} 1-\mathrm{Mo}-\mathrm{C} 3 & 85.4(4) & \mathrm{C} 2-\mathrm{Mo}-\mathrm{C} 3 & 81.1(4) \\ \mathrm{O} 4-\mathrm{Mo}-\mathrm{C} 1 & 99.4(1) & \mathrm{O} 4-\mathrm{Mo}-\mathrm{C} 2 & 101.2(1) \\ \mathrm{O} 4-\mathrm{Mo}-\mathrm{C} 3 & 171.6(3) & \mathrm{O} 5-\mathrm{Mo}-\mathrm{C} 1 & 96.3(3) \\ \mathrm{O} 5-\mathrm{Mo}-\mathrm{C} 2 & 175.8(3) & \mathrm{O} 5-\mathrm{Mo}-\mathrm{C} 3 & 103.1(3) \\ \mathrm{O} 7-\mathrm{Mo}-\mathrm{C} 1 & 176.8(3) & \mathrm{O} 7-\mathrm{Mo}-\mathrm{C} 2 & 99.5(3) \\ \mathrm{O} 7-\mathrm{Mo}-\mathrm{C} 3 & 96.5(3) & & \end{array}$

